\title{
Étude de suivi des adolescentes migrantes employées de maison ayant participé à la première cohorte du programme Filles éveillées
}

Sarah Engebretsen

Population Council

Follow this and additional works at: https://knowledgecommons.popcouncil.org/departments_sbsr-pgy

Part of the Demography, Population, and Ecology Commons, Family, Life Course, and Society Commons, Gender and Sexuality Commons, International Public Health Commons, Medicine and Health Commons, and the Migration Studies Commons How does access to this work benefit you? Let us know!

\section{Recommended Citation}

Engebretsen, Sarah. 2013. "Étude de suivi des adolescentes migrantes employées de maison ayant participé à la première cohorte du programme Filles éveillées," Rapport technique. New York: Population Council. 


\section{ÉTUDE DE SUIVI DES ADOLESCENTES MIGRANTES \\ EMPLOYÉES DE MAISON AYANT PARTICIPÉ À LA PREMIËRE COHORTE DU PROGRAMME FILLES ÉVEILLEEES}

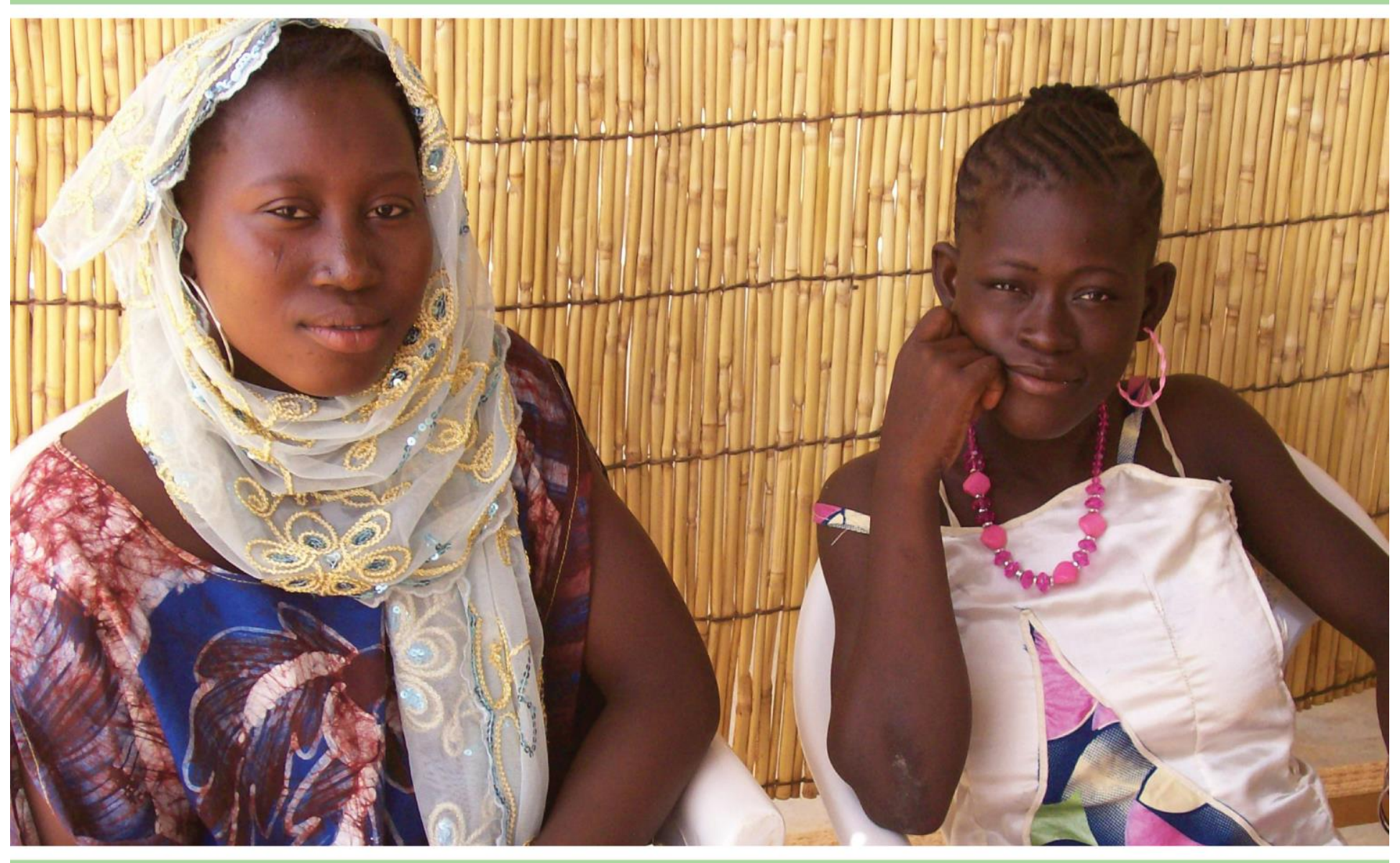

SARAH ENGEBRETSEN 


\section{(2) Population Council}

Le Population Council s'attelle aux questions cruciales de la santé et du développement qu'il s'agisse d'endiguer la progression du VIH, d'améliorer la santé reproductive et d'offrir aux jeunes la promesse d'une vie satisfaisante et productive. Par ses travaux de recherche en biomédecine, en sciences sociales et en santé publique dans 50 pays, il s'efforce d'apporter avec ses partenaires des solutions qui conduisent à des politiques, des programmes et des technologies plus efficaces, pour améliorer la vie partout dans le monde. Fondé en 1952, le Population Council est une organisation non gouvernementale à but non lucratif. II siège à New York sous la conduite d'un conseil d'administration international.

Population Council

One Dag Hammarskjold Plaza

New York, NY 10017

www.popcouncil.org

Indication de source : Engebretsen, Sarah. 2013. "Étude de suivi des adolescentes migrantes employées de maison ayant participé à la première cohorte du programme Filles éveillées. " New York : Population Council.

Photo courtoisement offerte par le Population Council.

(C) 2013 The Population Council, Inc. 


\section{INTRODUCTION}

La migration circulaire (du milieu rural au milieu urbain et retour), courante parmi les adolescents et adolescentes d'Afrique occidentale, est perçue tel un rite de passage pour les garçons comme pour les filles (Temin et al. 2013). La motivation de la migration est plurielle, mais elle répond avant tout à la quête de débouchés économiques. Ainsi le travail domestique pour les adolescentes migrantes. II s'agirait, selon les estimations de l'OIT, de la principale occupation des filles de moins de 16 ans à l'échelle mondiale (Temin et al. 2013). La base factuelle de données relatives à la migration et au travail domestique des jeunes est cependant plutôt maigre, entravant dès lors l'établissement de priorités d'allocation de ressources et de programmation et la mise à l'ordre du jour de la protection et de la promotion de la santé et du bien-être adolescents. Les données d'efficacité programmatique disponibles s'interrompent généralement au moment de la conclusion des programmes, sans suivi des adolescents à plus longue échéance et partant, sans capacité d'évaluation intégrale des effets durables des programmes conçus à l'intention des populations adolescentes mobiles.

Cette étude suit un sous-échantillon d'adolescentes migrantes employées de maison au Burkina Faso après leur participation à la première cohorte du programme pilote Filles éveillées. Ce programme a été conçu pour offrir aux filles migrantes employées de maison l'occasion de renforcer leurs réseaux sociaux et de développer leurs compétences nécessaires à la vie adulte. Sur une période de 30 semaines, les participantes ont assisté à des rencontres de groupe hebdomadaires sous la conduite de femmes mentors, dans le but d'acquérir et développer leurs compétences de vie courante et de santé (y compris sexuelle et reproductive), ainsi que leurs capacités financières. Le plan d'intervention, les critères de sélection des participantes, le recrutement, les qualifications des mentors et leur formation sont décrits plus avant dans un rapport publié sur la première cohorte du programme (Engebretsen 2012). L'Association Tié a assuré la mise en œuvre de la première cohorte de Filles éveillées en 2011-2012, auprès d'adolescentes âgées de 11 à 16 ans. Des enquêtes de type pré- et post-test ont été menées auprès des participantes, dont un sous-échantillon a également été interviewé un an après la conclusion du programme. 


\section{MÉTHODES}

Le Population Council a mené une enquête pré- puis post-test auprès des adolescentes migrantes employées de maison de Bobo-Dioulasso membres de la première cohorte de participantes au programme Filles éveillées mené sur une période de huit mois $(n=180)$. Une enquête de suivi a effectuée auprès d'un souséchantillon de participantes en juin 2013, soit une année après la conclusion du programme. Le sous-échantillon se compose de filles qui vivaient toujours sur les lieux de mise en œuvre du programme (Diarradougou, Koko, Sikassocira, Accart-Ville, Sarfalao et Lafiabougou) et qui ont accepté de participer à l'entretien un an plus tard $(n=50)$. Cette analyse compare un sous-échantillon $(n=50)$ des participantes à la première cohorte du programme Filles éveillées ayant répondu à l'enquête finale de 2012 aux filles interviewées lors de l'entretien de suivi en 2013. ${ }^{1}$ L'analyse a été conçue dans le but d'évaluer la durabilité de la connaissance, des attitudes et des comportements des filles sur les points clés du programme.

Les filles migrantes comprises dans la tranche d'âge considérée ${ }^{2}$ et qui étaient employées de maison sur l'un des sites de projet sélectionnés ont été invitées à participer au programme. L'autorisation de leurs employeurs ou gardiens a été demandée. Les participantes et leurs employeurs ont été invités à signer le formulaire d'autorisation de participation au programme et le consentement à la participation des filles aux enquêtes afférentes. L'enquête, élaborée par l'équipe de recherche du Population Council, se composait principalement de questions de type fermé, concernant les caractéristiques sociodémographiques, les compétences de vie et le capital social, la santé et l'hygiène, la santé sexuelle et reproductive et les capacités financières. Les questions de l'enquête de suivi reflètent celles posées lors de l'enquête finale de 2012.

Étant donné la nature plutôt sensible des sujets abordés, les entretiens ont été menés exclusivement par des femmes et les questions ont été posées en dioula, la langue locale parlée à Bobo-Dioulasso. Les entretiens ont été menés en privé, à l'écart des employeurs et autres membres du ménage, pour que les filles puissent répondre à l'aise aux questions. Ils ont été planifiés au moment qui convenait le mieux aux filles, pour une durée de 45 minutes à une heure. Conscientes de l'emploi du temps chargé des filles, les intervieweuses ont planifié leurs entretiens en

\footnotetext{
1 Des 50 résidentes de Bobo-Dioulasso ayant accepté de se soumettre à l'entretien un an après la conclusion du programme, 41 avaient participé à l'enquête finale (les neuf autres étaient retournées dans leur village pour aider aux travaux des champs pendant la saison des pluies et étaient ensuite revenues à la ville). L'analyse comprend donc 41 paires assorties. Nous avons pour le reste sélectionné aléatoirement neuf autres participantes à l'enquête finale de 2012, de manière à disposer d'échantillons de 50 filles à chaque point temporel d'analyse.

2 La recherche formative a révélé que les adolescentes migrent par étapes, vers une petite ville d'abord, puis vers la capitale. Les âges ciblés diffèrent donc entre Bobo-Dioulasso (11 à 16 ans) et Ouagadougou (15 à 19 ans), où le programme a été mis en œuvre dans le cadre de la seconde cohorte.
} 
conséquence. La description complète des qualifications et de la formation des intervieweuses est décrite ailleurs (Engebretsen 2012).

La collecte des données a été effectuée sous la supervision d'un consultant expert dans le domaine de la recherche sur le terrain. La formation des intervieweuses a également été confiée à ce consultant, de même que la saisie et le nettoyage des données et que leur analyse, en collaboration avec un chercheur du Population Council. Un superviseur local a assuré le contrôle de qualité des données et la soumission de questionnaires complets. Les données ont été analysées sous la version SPSS 20.

Des variables composées ont été définies pour mesurer le capital social et la confiance en soi. Le capital social a été évalué par la mesure des éléments suivants : participation à des groupes autres que Filles éveillées, amitiés, disponibilité d'un lieu de rencontre avec ses amies, disponibilité d'un endroit où passer la nuit en cas de nécessité, connaissance d'une personne à qui emprunter de l'argent en cas d'urgence et connaissance d'une personne à qui demander conseil. Les variables ont été codées 0 ou 1 et additionnées, pour une étendue possible de 0-6 concernant la mesure du capital social. Une cote de 0 à 1 est signe de capital social limité ; de 2 à 3 , d'un capital moyen ; et de 4 à 6 , d'un capital social élevé. Les variables de confiance en soi ont été codées 0 ou 1 et additionnées, pour une étendue possible de 0-8. Les variables suivantes sont considérées : bonne perception de son pouvoir personnel, ${ }^{3}$ absence de timidité en présence d'autrui, capacité de prendre ses propres décisions, capacité de résister à la pression des pairs, satisfaction du niveau d'éducation atteint, espoir pour l'avenir, absence de sentiment de faiblesse ou de vulnérabilité et capacité de s'exprimer.

Cette analyse comprend une comparaison des moyennes et pourcentages de l'enquête finale effectuée au terme du programme de huit mois avec ceux de l'enquête de suivi, un an plus tard. Étant donné la taille réduite de l'échantillon, l'analyse n'évalue pas la signification statistique.

\section{RÉSULTATS}

\section{Caractéristiques de l'échantillon}

Les participantes à l'enquête de suivi étaient âgées de 12 à 18 ans. L'âge moyen des 50 filles ayant répondu à l'enquête de suivi était de 15,3 ans ; 82\% de ces filles ont déclaré vivre avec leurs employeurs. Aucune des filles n'a déclaré être mariée, en

\footnotetext{
${ }^{3}$ Une échelle à neuf échelons a été présentée aux répondantes, selon les différents niveaux de perception de pouvoir ( 0 =aucun pouvoir, 9 =pouvoir élevé). Une étendue de 0-4 a été codée comme faible perception de pouvoir, et de 5-9 comme perception élevée.
} 
conformité avec les résultats de l'enquête EDS, selon lesquels 2,5\% seulement des résidentes urbaines de 20 à 24 ans sont mariées avant l'âge de 15 ans (Population Council 2009). Six pour cent étaient fiancées ou promises. Plus de la moitié n'avaient jamais été scolarisées et, de celles qui l'avaient été, $6 \%$ seulement avaient achevé le cycle primaire. D'après les résultats d'un petit test d'alphabétisation, plus de trois quarts des filles ne savaient pas lire, $20 \%$ lisaient avec difficulté et $2 \%$ seulement lisaient parfaitement. Quatre filles sur cinq ont déclaré avoir un document d'identité personnel, probablement obtenu lors de la remise de cartes d'identification dans le cadre du programme Filles éveillées.

\section{Capital social et compétences de vie}

Reconnaissant le double désavantage des filles migrantes fraîchement arrivées à la ville, concernant les ressources qui leur sont disponibles et la construction de réseaux sociaux aptes à leur y donner accès (Temin et al. 2013), Filles éveillées leur a donné l'occasion d'étendre leurs réseaux en participant à des groupes de même sexe animés par des mentors féminines adultes. Une amélioration du capital social a été observée après la réalisation du programme, $42 \%$ des répondantes à l'enquête finale faisant état d'un capital social élevé, de même que $58 \%$ au suivi. La cote moyenne de capital social est aussi passée de 3,12 à l'enquête finale à 3,56 à celle de suivi.

Concernant la variable composée de confiance en soi, la cote moyenne a augmenté entre l'enquête finale et celle de suivi $(4,20$ à 4,70$)$. Six des huit variables de confiance en soi individuelles sont en hausse, comme illustré à la Figure 1. Les deux variables de confiance en soi qui n'ont pas changé entre l'enquête finale et le suivi sont l'absence de timidité en présence d'autrui et la capacité de s'exprimer. 
FIGURE 1: Mesures de confiance en soi, suivant le moment de l'enquête

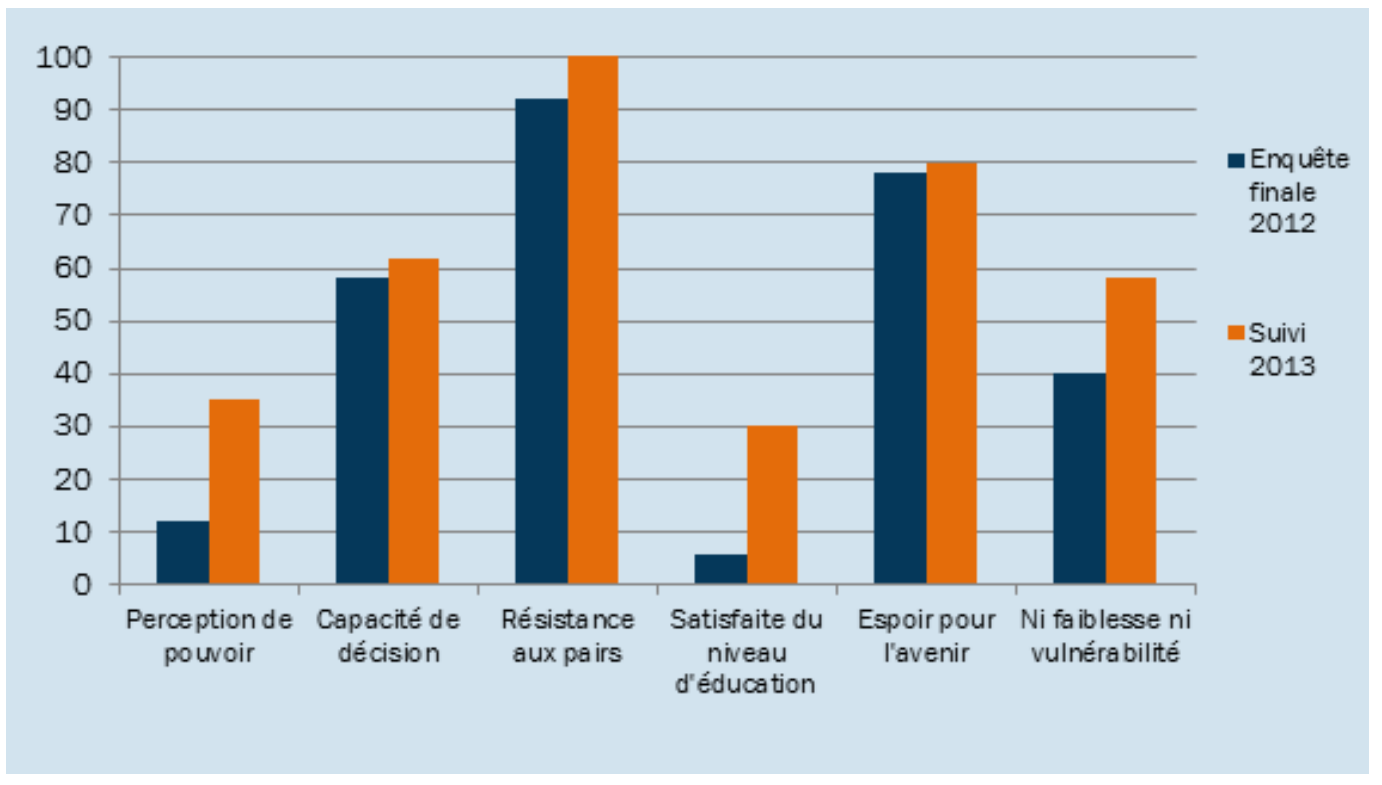

\section{Santé, y compris sexuelle et reproductive}

Filles éveillées a donné à ses participantes l'occasion de s'informer sur d'importantes questions de santé et d'hygiène. Le programme a aussi apporté aux filles des compétences qui leur permettent de gérer activement leur propre santé et exploré les attitudes relatives aux comportements sains. La capacité des filles à reconnaître les symptômes de maladies courantes s'est améliorée au fil du temps, de même que la sensibilisation au fait que les femmes enceintes doivent obtenir des soins prénatals et que les filles peuvent s'auto-examiner les seins, comme indiqué au Tableau 1. Les répondantes ont été interrogées sur les raisons pour lesquelles les filles de leur âge se rendent dans les centres de santé. Plusieurs réponses étaient possibles : pour obtenir une information sur la santé, la contraception et des tests de santé, notamment. Une amélioration de la sensibilisation aux raisons d'obtenir des soins de santé est également observée entre l'enquête finale et celle de suivi.

\section{TABLEAU 1: Pourcentages et répartition moyenne de la} connaissance des filles interrogées sur les questions de santé, suivant le moment de l'enquête

\begin{tabular}{|l|l|l|}
\hline & Fin & Suivi \\
\hline Reconnaissance des symptômes de maladies courantes & $48 \%$ & $74 \%$ \\
\hline $\begin{array}{l}\text { Sensibilisation au besoin de soins prénatals des } \\
\text { femmes enceintes }\end{array}$ & $48 \%$ & $66 \%$ \\
\hline
\end{tabular}




\begin{tabular}{|l|l|l|}
\hline $\begin{array}{l}\text { Sensibilisation au fait que les filles peuvent s'auto- } \\
\text { examiner les seins }\end{array}$ & $61 \%$ & $64 \%$ \\
\hline $\begin{array}{l}\text { Nombre moyen de raisons citées pour lesquelles les } \\
\text { adolescentes se rendent dans les centres de santé }\end{array}$ & 1,38 & 2,28 \\
\hline
\end{tabular}

Les changements observés au niveau de la connaissance relative à la grossesse ne sont pas constants. De l'enquête finale au suivi, on enregistre une augmentation du pourcentage de filles déclarant que l'absence de règles peut être signe de grossesse (30\% vs $62 \%$ ). Les répondantes ont été invitées à nommer les symptômes de la grossesse. Leur connaissance a été interprétée comme limitée (0-1 symptôme), moyenne (2-4 symptômes) ou élevée (au moins 5 symptômes). Le pourcentage de répondantes classées dans la catégorie de connaissance élevée a doublé entre l'enquête finale et celle de suivi ( $4 \%$ vs $8 \%$ ). Malgré ces gains, on n'observe toutefois aucun changement au niveau de la sensibilisation à la nécessité de soins prénatals pour les femmes enceintes. On observe du reste un amoindrissement entre les deux enquêtes en ce qui concerne la connaissance du moment du cycle menstruel où la femme est féconde et de celle de la possibilité de se retrouver enceinte après un rapport sexuel non protégé ( $36 \%$ vs $26 \%$ et $84 \%$ vs $60 \%$, respectivement).

On observe des changements au niveau de la connaissance de la planification familiale, mais pas toujours dans la direction attendue. Les filles ont été invitées à nommer toutes les méthodes de planification familiale dont elles avaient conscience. Le nombre moyen de méthodes citées diminue entre les deux enquêtes, de 2,18 à 1,64. Le pourcentage de filles sensibilisées aux méthodes de planification familiale traditionnelles augmente entre l'enquête finale et celle de suivi (44\% vs $68 \%$ ), alors que celui des filles informées d'une méthode moderne quelconque - pilule, injectable, stérilet ou préservatif - diminue en l'espace de l'année écoulée (de 82\% vs $50 \%$ ). Le pourcentage de répondantes informées sur les prestataires officiels de la planification familiale au sein de la communauté est aussi en baisse entre l'enquête finale et celle de suivi ( $76 \%$ vs $46 \%)$.

Les répondantes ont été invitées à répondre à une série de questions relatives aux infections sexuellement transmissibles, y compris le $\mathrm{VIH}$, et des mesures sommaires ont été définies pour révéler la connaissance moyenne. Les résultats entre l'enquête finale et celle de suivi paraissent contradictoires, comme indiqué au Tableau II. On notera cependant que le pourcentage de filles conscientes du fait que le mariage ne protège pas contre le VIH s'est amélioré entre les deux enquêtes (64\% vs $78 \%$, non indiqué). 
TABLEAU 2: Répartition moyenne de la connaissance des filles interrogées sur les IST et le VIH, suivant le moment de l'enquête

\begin{tabular}{|l|c|c|}
\hline \multicolumn{2}{|l|}{ Fin } & Suivi \\
\hline Nombre moyen de symptômes d'IST identifiés & 1,34 & 1,02 \\
\hline $\begin{array}{l}\text { Nombre moyen de méthodes de protection personnelle } \\
\text { citées contre le VIH }\end{array}$ & 1,46 & 1,36 \\
\hline $\begin{array}{l}\text { Nombre moyen de lieux de dépistage du VIH identifiés } \\
\text { dans la communauté }\end{array}$ & 1,00 & 1,06 \\
\hline
\end{tabular}

\section{Capacités financières}

Comme les adolescentes migrantes employées de maison sont économiquement actives, Filles éveillées a cherché à renforcer leurs capacités d'épargne, en mettant tout spécialement l'accent sur la budgétisation, l'épargne, la planification de l'avenir et l'aptitude à parler de questions d'argent. En dépit du fait que plus de la moitié des participantes au programme n'avaient jamais été scolarisées, leurs capacités de calcul étaient supérieures à celles attendues, 96\% ayant répondu correctement à une question d'addition en 2012.

Les déclarations relatives aux principaux objets de dépense restent constantes entre l'enquête finale et celle de suivi : il s'agit notamment de produits de beauté, de vêtements et d'envois d'argent à la famille. Au moment de l'enquête finale, deux tiers des filles déclaraient décider seules de la manière de dépenser leur argent. Cette proportion reste inchangée au suivi.

Aux deux enquêtes, quatre filles sur cinq déclarent épargner leur argent, régulièrement (chaque jour, chaque semaine ou chaque mois) pour plus de la moitié. À la question de savoir pourquoi elles épargnent, les filles donnent des raisons à court aussi bien qu'à long terme. Les raisons d'épargne à court terme se définissent selon ce que les filles entendent faire de leur argent dans les trois prochains mois : préparation à une situation d'urgence, achats personnels, achat de vêtements ou d'ustensiles, ou satisfaction d'autres besoins de base, notamment. La motivation à long terme couvre les raisons d'épargne en vue de dépenses éventuelles durant l'année à venir : pour le paiement de soins de santé, l'envoi d'argent à la famille, la préparation au mariage, l'achat de fournitures nécessaires aux activités productives ou les frais de formation professionnelle, par exemple. Une faible hausse se révèle entre l'enquête finale et celle de suivi au niveau de la proportion de filles épargnant à court terme ( $58 \%$ vs $60 \%$ ) comme à long terme ( $24 \%$ vs $26 \%$ ). La connaissance des mécanismes d'épargne formels s'améliore entre 2012 et 2013 (8\% vs 14\%). La proportion de filles déclarant avoir un objectif d'épargne à court terme demeure constante d'une enquête à l'autre, à un peu moins de la moitié des répondantes. 


\section{DISCUSSION}

Le programme Filles éveillées a été mis au point dans le but d'accroître les réseaux sociaux et de développer les compétences des adolescentes migrantes employées de maison au Burkina Faso urbain. Bien qu'il existe d'autres programmes au service de cette population, Filles éveillées est l'un des premiers à viser le renforcement des compétences de santé, sociales et économiques des filles avant que ne survienne un malheur dans leur vie. Le programme s'est déroulé sur deux cycles pilotes : le premier en 2011-2012 à Bobo-Dioulasso, puis le second en 2012-2013 à Ouagadougou et Bobo-Dioulasso. Cette analyse de suivi nous a donné une rare occasion de suivre les filles qui avaient participé à la première cohorte de l'intervention et d'évaluer ainsi l'évolution éventuelle de leur connaissance, de leurs attitudes et de leurs comportements sur les points clés du programme un an après sa conclusion. On notera qu'une évaluation de processus avait été réalisée entre les deux cycles, afin d'évaluer l'efficacité du programme et de suggérer les améliorations possibles à la structure, à la mise en œuvre et au contenu avant le lancement de la seconde cohorte. Les adaptations programmatiques résultant de cette évaluation ont vraisemblablement contribué aux changements observés au niveau de la seconde cohorte. $^{4}$

Dans l'ensemble, les observations laissent entendre une certaine évolution positive de la connaissance, des attitudes et des comportements chez les participantes au programme un an après sa conclusion. Les conclusions donnent à penser que des effets durables sont plus susceptibles d'être observés au niveau du capital social, de la confiance en soi et de la sensibilisation aux questions générales de santé qu'à celui des autres domaines d'intérêt. L'évolution de la connaissance en matière de santé sexuelle et reproductive et des comportements financiers n'est pas constante et se produit même à rebours dans certains cas.

Cette étude présente plusieurs limites. D'abord, étant donné la taille réduite de l'échantillon, l'analyse omet la perspective de la signification statistique. Ensuite, l'absence de témoins limite la capacité de conclure que les changements observés entre l'enquête finale et celle de suivi sont effectivement dus à l'intervention. Les itérations futures du programme bénéficieront certes d'un plan de recherche plus rigoreux. De même, les participantes à l'enquête de suivi formaient un échantillon volontaire de filles qui avaient choisi de continuer à vivre en milieu urbain plutôt que de retourner dans leurs villages, d'où le risque d'un biais de sélection. Certains des changements positifs observés d'une enquête à l'autre ne tiennent dès lors peut-être 4 Voir Engebretsen (2013) pour une description des ajustements apportés entre les deux cohortes et pour
une évaluation de la seconde cohorte. 
pas aux effets à long terme du programme, mais plutôt à une meilleure assimilation à la vie en milieu urbain. Enfin, cette analyse compare les mesures finales, et non initiales, avec celles de suivi, diluant peut-être ainsi les effets observés dans le temps.

Cela dit, l'analyse présente une perspective importante sur la manière dont les adolescentes migrantes s'intègrent dans le milieu urbain après leur période d'installation initiale. Les changements non convergents de la connaissance en matière de santé sexuelle et reproductive ou, dans certains cas, le recul observé entre l'enquête finale et celle de suivi démontrent que la connaissance ne se conserve pas toujours sur le long terme et qu'il importe de continuer à atteindre cette population par l'apport de formations de " recyclage ". L'équipe chargée du projet tient déjà compte de ces considérations pour les cohortes à venir et a mis au point une " boîte à outils " destinées aux anciennes participantes. Le but en est de rappeler aux filles les leçons clés du programme et de les aider à transmettre cette information à d'autres adolescentes, dans leurs communautés urbaines ou de retour dans leurs villages.

II n'est plus à démontrer que le capital social aide les filles migrantes dans leur transition vers la vie urbaine (Temin et al. 2013) et que des programmes explicitement axés sur le renforcement de ce capital, de la confiance personnelle et des compétences des filles fraîchement arrivées sont nécessaires. Filles éveillées a montré qu'il est possible de renforcer le capital social et la confiance en soi des filles migrantes et que ces concepts - en particulier les variables relatives à la perception de soi - peuvent être maintenus ou même renforcés une année après l'achèvement du programme. Une recherche complémentaire doit être menée pour tester les effets à long terme du capital social et de la confiance personnelle des filles migrantes sur la connaissance, les attitudes et les comportements. Les conclusions de cette analyse établissent clairement aussi la nécessité d'évaluations complémentaires des programmes conçus à l'intention des adolescentes migrantes employées de maison, ainsi que de nouvelles études longitudinales concernant les implications de la migration sur le bilan social, économique et de santé des filles.

\section{RÉFÉRENCES}

Engebretsen, S. 2012. Observations initiales et finales de Filles Éveillées: Programme pilote de renforcement des competences des adolescents migrantes employees de maison. Cohorte 1 (2011-2012), Bobo-Dioulasso. New York: Population Council.

Engebretsen, Sarah. 2013. "Évaluation de Filles éveillées : Programme pilote pour adolescentes migrantes employées de maison. „ New York : Population Council 
Population Council. 2009. " Etude approfondie sur les adolescents : Exploiter les données pour identifier et atteindre les jeunes les plus vulnérables - Burkina Faso 2003." New York.

Temin, M., M.R. Montgomery, S. Engebretsen, et K.M. Barker. 2013. Girls on the Move: Adolescent Girls and Migration in the Developing World. New York: Population Council. 